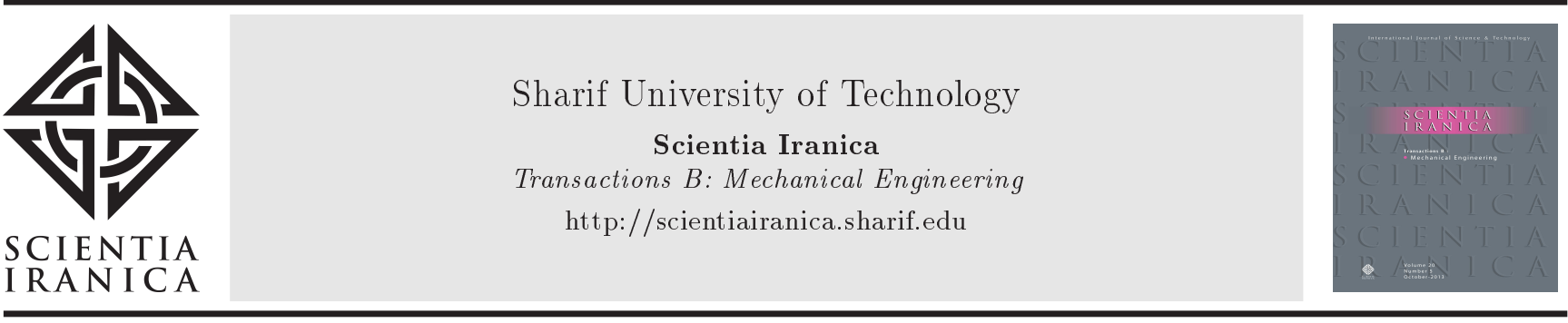

\title{
The study of cavitation phenomenon in multistage centrifugal pump and reduction of its damages
}

\author{
B. Ranjbar*, M. Ehghaghi, and F. Ranjbar \\ Department of Mechanical Engineering, University of Tabriz, Tabriz, postcode: 5163843560, Iran
}

Received 4 November 2018; received in revised form 15 November 2018; accepted 18 February 2019

\author{
KEYWORDS \\ Centrifugal pump; \\ Cavitation; \\ API standard; \\ CFD; \\ NPSH; \\ Damage.
}

\begin{abstract}
Cavitation phenomenon in centrifugal pumps is the main cause of failures in pump components such as impeller and volute. To evaluate this phenomenon, first of all, the flow field in a BB2 API multistage centrifugal pump with and without cavitation is studied. Additionally, to improve the impeller inlet condition and reduction of cavitation possibility, Stepannof and Dixon theory is used. This study mainly focuses on the concept of cavitation in pump, pump performance curve, system pump curve, and Net Positive Suction Head (NPSH). The main objective of this research is to determine the best operating pump range. It is interesting to investigate the system pump curve prediction to identify the inception cavitation zone. Therefore, a theoretical system pump curve was generated using Microsoft Excel 2010; in addition, Catia V5 R21 and ANSYS CFX 14 are applied to create a computational fluid dynamic model from simulation results; the reduction of NPSHa values leads to the onset of cavitation. The major findings of this paper present the theoretical and numerical results concerning the pump characteristics and performance breakdown in different flow conditions. Therefore, the best operating pump range is identified at a flow rate of $330 \mathrm{~m}^{3} / \mathrm{hr}$ to avoid the occurrence of cavitation in pumps.
\end{abstract}

(C) 2020 Sharif University of Technology. All rights reserved.

\section{Introduction}

In recent years, with the advancement of the analytical tools of fluid dynamics, it has become possible to model and study cavitation phenomenon numerically. In addition to providing a better understanding of the nature of this phenomenon, it is also possible to compare and validate the results of experimental and numerical data based on existing theories available [1$3]$. In recent years and decades, researchers have made notable attempts to study cavitation in various types of centrifugal pumps, both experimentally and numerically; this paper focuses on some of the most important pumps [4,5]. In [6], a model called Full Cavitation

\footnotetext{
*. Corresponding author.

E-mail address: beh.ranjbar.mc@gmail.com (B. Ranjbar)
}

doi: $10.24200 /$ sci. 2019.52112 .2539 was used to predict the performance of a centrifugal pump with fluid flow under cavitation conditions. In this model, a steam transfer equation for the steam phase is coupled with Naviertox coupling turbulence equations, and Riley-Polest equations are used. The effects of turbulence fluctuations and incompressible gases were also implemented to complete the proposed model. In [7], the cavitation flow was analyzed in a lowspeed centrifugal pump using two Computational Fluid Dynamics (CFD) trading codes, and the results were compared with those of the performed experiments. The findings showed that the cavitation bubbles appeared in the middle arc of the suction surface, where the static pressure is at its lowest level. Bubbles are developed in the downstream and, when they reach the nose, a wedge that resembles a hole in the neck and a suppressive surface, the cavitation bubbles appear under pressure at the edge of the attack. In [4], the numerical and experimental analysis of the cavitation 
phenomenon in a centrifugal pump was carried out. An experimental study of the hydraulic circuit of the centrifugal pump test with special features was conducted for monitoring the flow between the pump blades and numerical analysis of the phenomenon by means of flow software as a flow analysis of the pump under single-phase, three-dimensional, turbulent, and slippery conditions with a blade in different working conditions. Using a pressure distribution, we detected areas of the cavitation region in different portions of the pump and compared the results with the experimental data. The results point out that the bubble appearance method is a convenient and accurate method for studying the cavitation phenomenon, and the MRF model in the FlowNet software is also a suitable apparatus for analyzing the flow of turbo-turbine engines with incompressible flows. In [8], the main objective was the numerical simulation of a Francis centrifugal pump turbine to evaluate its performance under cavitation conditions and, to this end, Riley-Polest's cavitation model and NPSHreq value from the numerical solution by Anticipated ANSYS CFX software were used and, then, compared with the results of experimental data. Li et al. [9] performed a numerical simulation of cavitation phenomenon and managed to present Net Positive Suction Head (NPSH) prediction for a PumpLinx Propeller Centrifugal Pump. The results showed that the pressure level at the cavitation inlet was always produced from the rear of the blade edge (where the bubbles form). Nevertheless, cavitation damage occurred in a downstream area where bubbles collapsed due to the pressure exerted on them.

The present paper examines the cavitation phenomenon in centrifugal pumps, which is mostly the most common fault in these types of pumps and can generate noise and vibrations at high levels, resulting in serious damage to the pump components and catastrophic failures. The objective of this study is to make a precise prediction of the starting point of cavitation and figure out how to distribute the pressure field and the volume of steam in the butterfly passages of a centrifuge pump and its setting criteria in order to prevent the development of cavitation, increase the life of the pump, and prevent system shutdown.

\section{Research method}

\subsection{Mathematical model of turmoil}

Since the cavitation phenomenon occurs at high Reynolds numbers, most of the circuits containing this flow phenomenon can become confusing to differentiate and, thus, turbulence modeling is required [10-13]. According to the studies, the $k-\varepsilon$ disturbance model for cavity flows has the highest compatibility with experimental results. Hence, this model is used to model disturbances. In this model, $k$ is the kinetic energy and defined as the variance of velocity, which has the dimension $\left[L^{2} T^{-2}\right]$. Herein, $\varepsilon$ is the turbulent eddy distribution rate (the rate at which velocity fluctuations are propagated) with dimensions $k$ in each time unit $\left[L^{2} T^{-3}\right]$. The $k-\varepsilon$ model introduces two new variables to the system of equations. As shown in previous estimates, the conjugation and momentum equations are given in Eqs. (1) and (2) [10,14]:

$$
\begin{aligned}
& \frac{\partial \rho}{\partial t}+\frac{\partial}{\partial x_{j}}\left(\rho U_{j}\right)=0 \\
& \frac{\partial \rho U i}{\partial t}+\frac{\partial}{\partial x_{j}}\left(\rho U_{i} U_{j}\right)= \\
& \quad-\frac{\partial \rho^{\prime}}{\partial x_{i}}+\frac{\partial}{\partial x_{j}}\left[\left(\frac{\partial U_{i}}{\partial x_{j}}+\frac{\partial U_{j}}{\partial x_{i}}\right)\right]+S_{M} .
\end{aligned}
$$

The $k-\varepsilon$ model is based on the concept of edible viscosity. Therefore, Eq. (3) is given for the equivalent viscosity:

$$
\mu_{e f f}=\mu+\mu_{t}
$$

where $\mu_{t}$ is the turbulence viscosity. This model assumes that the turbulence viscosity with turbulent kinetic energy and its distribution rate are linked through Eq. (4):

$$
\mu_{t}=C_{\mu} \rho \frac{k^{2}}{s}
$$

where $C_{\mu}$ is a value. The values of $k$ and $\varepsilon$ are determined directly through the transport equations (Eqs. (5) and (6)):

$$
\begin{aligned}
\frac{\partial(\rho k)}{\partial t} & +\frac{\partial}{\partial x_{j}}\left(\rho U_{j} k\right)=\frac{\partial}{\partial x_{j}}\left[\left(\mu+\frac{\mu_{t}}{\sigma_{k}}\right) \frac{\partial k}{\partial x_{j}}\right] \\
& +P_{k}-\rho \varepsilon+P_{k b}, \\
\frac{\partial(\rho \varepsilon)}{\partial t} & +\frac{\partial}{\partial x_{j}}\left(\rho U_{j} \varepsilon\right)=\frac{\partial}{\partial x_{j}}\left[\left(\mu+\frac{\mu_{t}}{\sigma_{\varepsilon}}\right) \frac{\partial \varepsilon}{\partial x_{j}}\right] \\
& +\frac{\varepsilon}{k}\left(C_{s 1} P_{k}-C_{s 2} \rho \varepsilon+C_{s 1} P_{k b}\right),
\end{aligned}
$$

where $C_{s_{2}}, C_{s_{1}}, \sigma_{\varepsilon}$, and $\sigma_{k}$, are constant coefficients, and $P_{k b}$ and $\rho_{\varepsilon}$ represent the effect of floating forces. $P_{k}$ is also the turbulence generated by the viscosity forces, modeled using Eq. (7):

$$
P_{k}=\mu_{t}\left(\frac{\partial U_{i}}{\partial x_{j}}+\frac{\partial U_{j}}{\partial x_{i}}\right) \frac{\partial U_{i}}{\partial x_{j}}-\frac{\partial U_{k}}{\partial x_{k}}\left(3 \mu t \frac{\partial U_{k}}{\partial x_{k}}\right)+\rho(k)
$$

For an incompressible flow, $\frac{\partial U_{k}}{\partial_{x_{k}}}$ is small. The second term on the right-hand side of the equation has no significant contribution to production. For compressible flows, $\frac{\partial U_{k}}{\partial x_{k}}$ is high only in areas with instant dying such as shocks. 
The most commonly used method for the discretization of governing equations, included into CFDs such as CFX $[15,16]$, is the finite volume method. In this method, the computational domain is divided into a number of volume controls to which survival rules are applied separately, thus ensuring the survival of proportional quantities such as mass, momentum, and energy in the elements and the entire computing range; yet, the volume method is entitled to few advantages. The FVM allows the application of unstructured networks, which greatly reduces the computational time.

\section{2. $C A D$ model}

The numerical model, shown in Figure 1, was built using the Blast Generation ANSYS-CFX Software Packet Center Impeller Modeling Tool. In this tool, the profile of the blade is performed based on input design parameters such as head, discharge, and rotational speed, which usually varies. Therefore, a reverse engineering technique was used to solve the problem. To this end, a 3D scanner device and the profile of one propeller blade were used (Figure 2). Further to that, following the production of cloud points by the device, they were converted to surface in design of catia software. Then, parts design is started and the model is generated as shown in Figure 3 . In

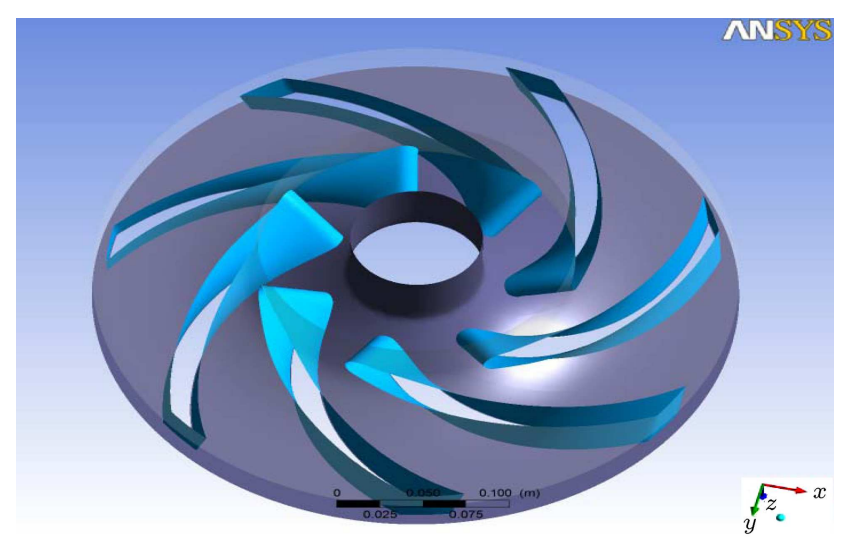

Figure 1. Modeling impeller in the blade generation environment. order to ensure greater compatibility between modeling permits in the blade generation environment with those used in laboratory work, the coordinates of the meridian of the blade were extracted from the modeling map and replaced by the blades generated by blade generation. The proposed model is characterized by a basic geometry so as to create a limited volume mesh.

Concerning the propagation of the impeller geometry, only one passage from the propeller, including a major model, is modeled. Figures 4 and 5 are shown the normal and improved meshed in sensitive points respectively.

\section{Results}

Table 1 shows the data sheet for the results of the pump test that consists of three major parts. The first part includes the measured data in which the corresponding parameters are recorded by the measurement tools, as described in the previous chapter. The second part contains information about the values that have been calculated using the relevant relationships. The third part includes the corrected data. As noted earlier, the speed of the test run, $\left[N^{*}\right]$, should be about $3 \%$ of the speed provided on the data sheet pump, $[N]$. Therefore, the results of the test must undergo a revision for the head, power, and flow in the tolerance range provided by ISO 9906A through Eq. (8) as the similarity rules:

$$
\begin{aligned}
& 0.97 \leq \frac{N^{*}}{N} \leq 1.03 ; \quad H^{*}=H \cdot\left[\frac{N^{*}}{N}\right]^{2} ; \\
& Q^{*}=Q \cdot\left[\frac{N^{*}}{N}\right] .
\end{aligned}
$$

By using the data sheet for pumps for non-cavitation flow, the pump characteristic curves can be plotted as in Figures 6-8. As expected, with an increase in discharge, the pump head is reduced and the pump efficiency becomes equal to that of the design point.

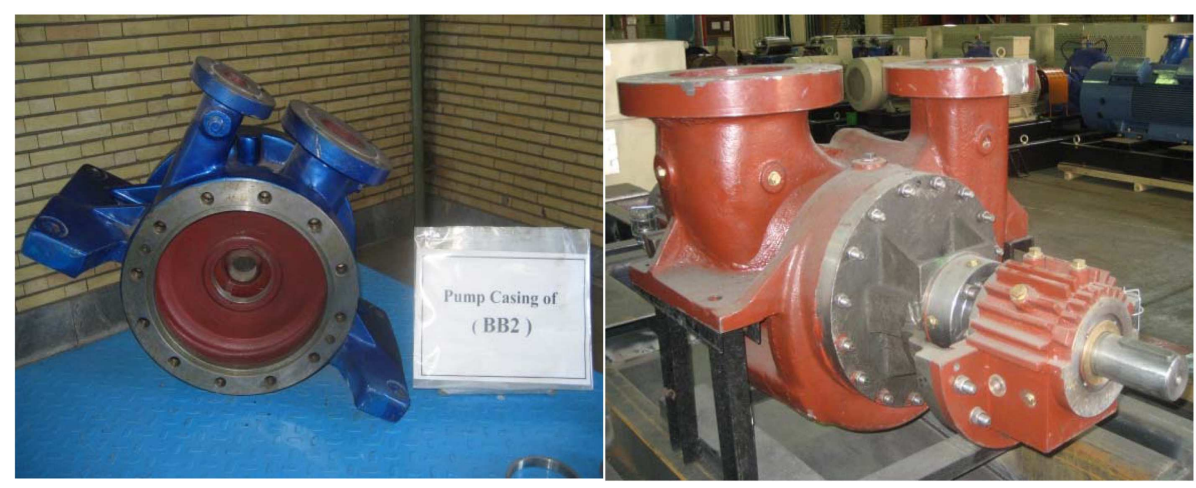

Figure 2. Sample of pumps in top-top configuration. 


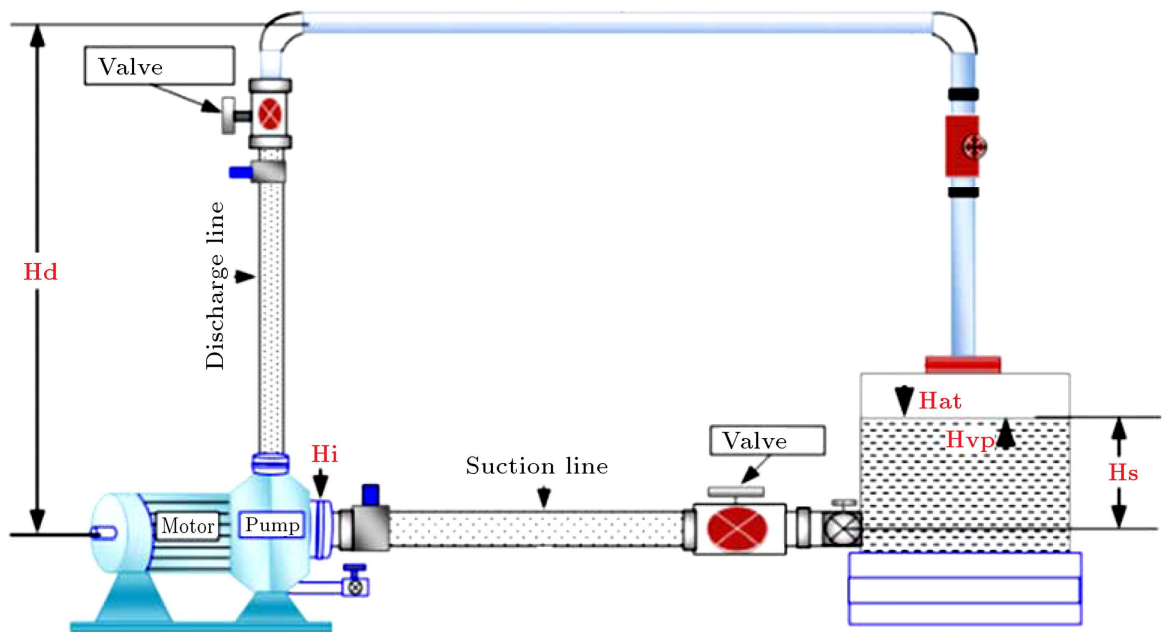

Figure 3. Schematic of a pump circle.

Table 1. Test results for non-cavitation flow.

\begin{tabular}{|c|c|c|c|c|c|c|c|c|}
\hline \multicolumn{9}{|c|}{ Pump specification } \\
\hline \multicolumn{2}{|l|}{ Type: WKL 150} & \multicolumn{4}{|c|}{$\begin{aligned} \text { Speed }(\mathrm{rpm}): & 1480 \\
\text { Diameter }(\mathrm{mm}): & 360 \mathrm{Imp}\end{aligned}$} & \multicolumn{3}{|c|}{ Power (kW): 200} \\
\hline \multicolumn{9}{|c|}{ Motor specification } \\
\hline \multicolumn{2}{|l|}{ Type: ABB } & \multicolumn{4}{|c|}{ Speed (rpm): 1483} & \multicolumn{3}{|c|}{ Current (kW): 200} \\
\hline \multicolumn{9}{|c|}{ Test condition } \\
\hline \multicolumn{2}{|l|}{ Gravity $\left(\mathrm{m} / \mathrm{s}^{2}\right): 8.9$} & \multicolumn{4}{|c|}{ Dedisity $\left(\mathrm{kg} / \mathrm{m}^{3}\right): 1000$} & \multicolumn{3}{|c|}{ Suction Pipe $(\mathrm{mm}): 200$} \\
\hline \multirow{2}{*}{\multicolumn{2}{|c|}{ Discharge pipe (mm): 150}} & \multirow{2}{*}{\multicolumn{4}{|c|}{$\begin{array}{c}\text { Suction gauge } \\
\text { height }(\mathrm{m}): 1.85\end{array}$}} & \multirow{2}{*}{\multicolumn{3}{|c|}{$\begin{array}{l}\text { Discharge gauge } \\
\text { height }(\mathrm{m}): 1.85\end{array}$}} \\
\hline & & & & & & & & \\
\hline \multicolumn{9}{|c|}{ Measured value } \\
\hline Test point & 1 & 2 & 3 & 4 & 5 & 6 & 7 & 8 \\
\hline Speed (rpm) & 1495 & 1493 & 1493 & 1493 & 1493 & 1493 & 1493 & 1493 \\
\hline Capacity $\left(\mathrm{m}^{3} / \mathrm{h}\right)$ & 97 & 150 & 199 & 240 & 280 & 330 & 385 & 452 \\
\hline Suction head (bar) & 0.21 & 0.21 & 0.20 & 0.20 & 0.20 & 0.19 & 0.17 & 0.11 \\
\hline Head discharge (bar) & 15.45 & 14.9 & 14.28 & 13.88 & 13.18 & 12.15 & 10.93 & 9.13 \\
\hline Abs current (A) & 257 & 265 & 280 & 293 & 305 & 322 & 338 & 355 \\
\hline Voltage (V) & 380 & 380 & 380 & 380 & 380 & 380 & 380 & 380 \\
\hline Abs power $(\mathrm{kW})$ & 97 & 106 & 118 & 129 & 139 & 151.5 & 162 & 175 \\
\hline \multicolumn{9}{|c|}{ Calculated data } \\
\hline Suction $\left(v^{2} / g\right)$ & 0.04 & 0.09 & 0.16 & 0.23 & 0.31 & 0.43 & 0.59 & 0.82 \\
\hline Discharge $\left(\mathrm{v}^{2} / \mathrm{g}\right)$ & 0.12 & 0.28 & 0.53 & 0.73 & 0.99 & 1.37 & 1.87 & 2.60 \\
\hline Total head (m) & 103.6 & 100 & 96 & 93.33 & 88.7 & 81.9 & 74 & 62.53 \\
\hline Power hydraulic (kW) & 41.1 & 61.3 & 78.1 & 91.6 & 101.5 & 110.5 & 116.5 & 116 \\
\hline Total efficiency $(\%)$ & 42.2 & 57.8 & 66.2 & 71 & 73 & 73.2 & 71.9 & 66.3 \\
\hline \multicolumn{9}{|c|}{ Corrected test } \\
\hline Speed (rpm) & 1450 & 1450 & 1450 & 1450 & 1450 & 1450 & 1450 & 1450 \\
\hline Capacity $\left(\mathrm{m}^{3} / \mathrm{h}\right)$ & 94.1 & 145.7 & 193.3 & 233.1 & 271.9 & 320.5 & 373.9 & 440.9 \\
\hline Total head (m) & 97.53 & 94.3 & 90.53 & 88.06 & 83.06 & 77.33 & 69.08 & 59 \\
\hline Aba. power $(\mathrm{kW})$ & 88.5 & 97.1 & 108.1 & 118.2 & 127.3 & 138.3 & 148.4 & 160.3 \\
\hline Power hydraulic (kW) & 37.2 & 56.2 & 71.5 & 83.9 & 93 & 101.3 & 106.7 & 106.3 \\
\hline Motor efficiency (\%) & 85 & 90 & 93 & 94 & 94 & 94 & 94 & 94 \\
\hline Pump efficiency (\%) & 49.9 & 64.3 & 71.1 & 75.5 & 77.7 & 77.9 & 76.9 & 70.5 \\
\hline
\end{tabular}




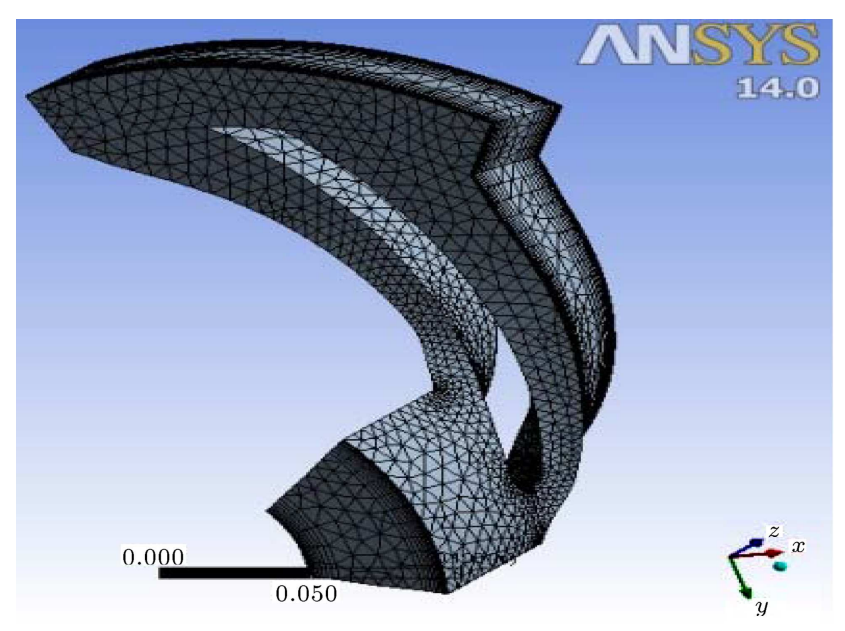

Figure 4. The geometric model of the meshing.

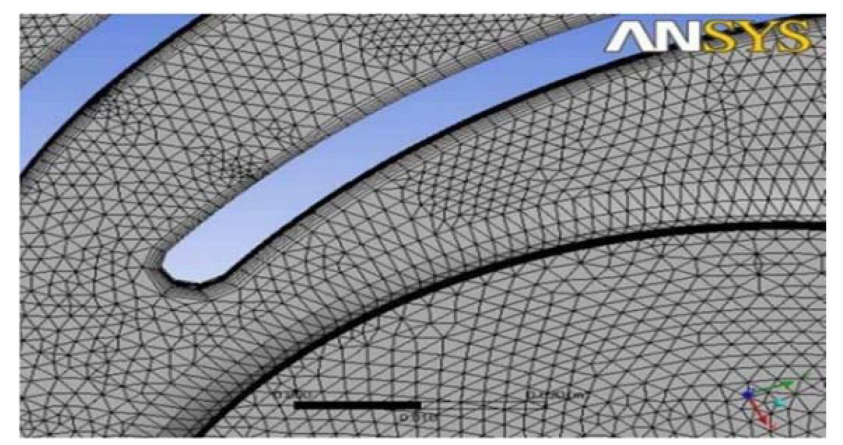

Figure 5. Improvement of the quality of the mesh around the surface of the blade and the non-layer layers.

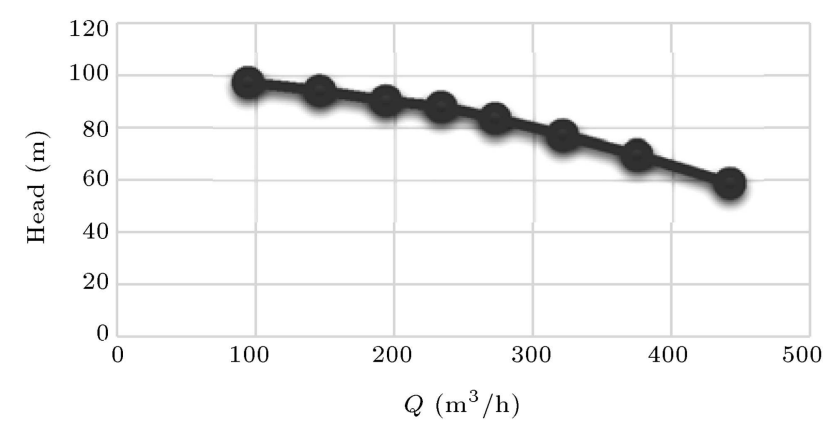

Figure 6. Experimental curve of head-flow.

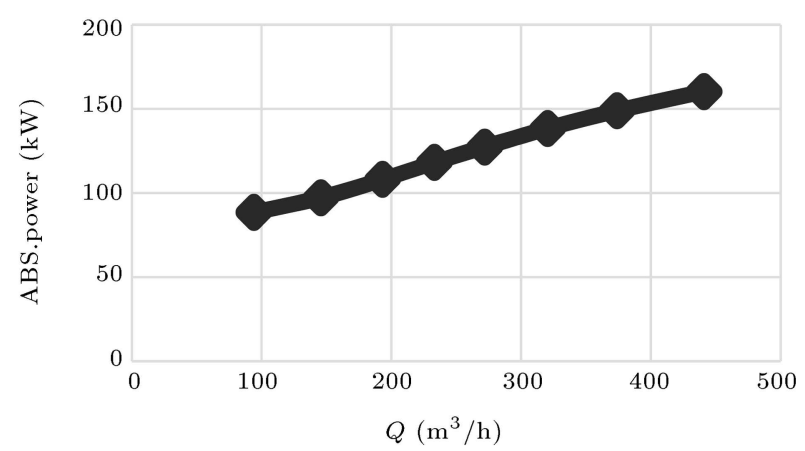

Figure 7. Power expeditionary curve-flow.

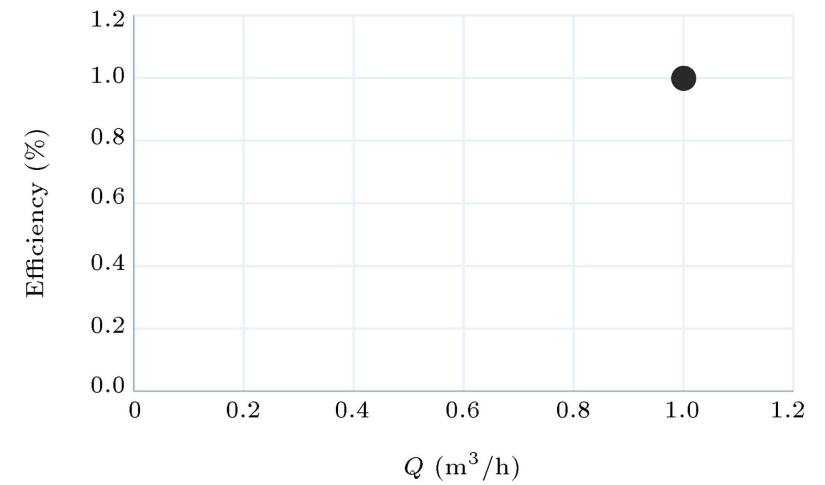

Figure 8. Experimental efficiency curve-flow.

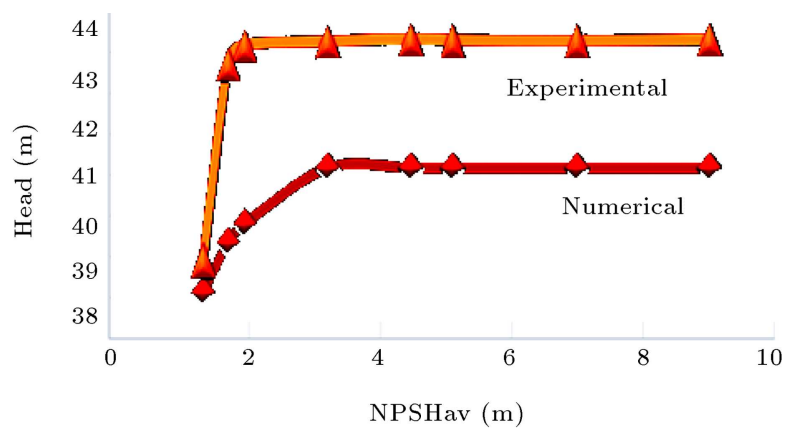

Figure 9. Comparison of experimental and numerical curves of H-NPSHav.

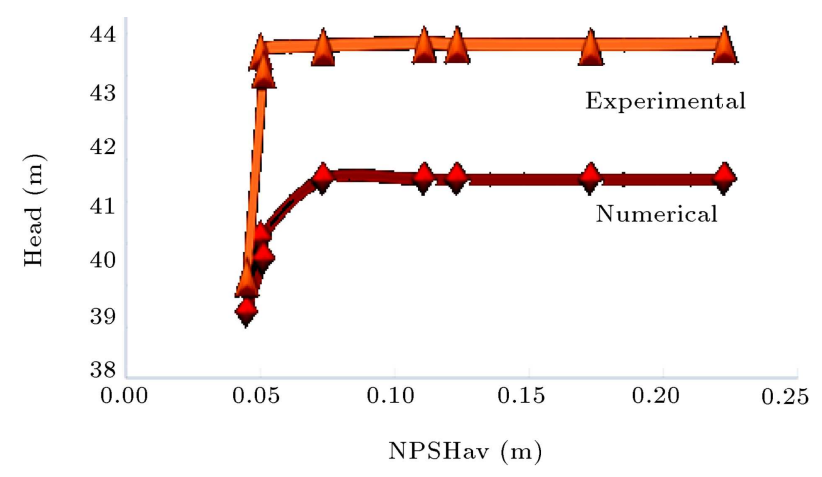

Figure 10. Comparison of experimental and numerical curves of H- $\sigma$ thoma.

\subsection{Comparison of experimental and numerical results}

Figures 9 and 10 show that the general head behavior with respect to the values of NPSH and $\sigma$ thoma is the same, which is why the determination of $i \sigma$ value is always important. On the other hand, the graphs represent the measured and simulated NPSHreq values in head loss of $3 \%$ and their good fit.

\subsection{Numerical simulation results}

3.2.1. Flow simulation without cavitation results Distribution of flow in the propellant

As shown in Figure 11, the pressure inside the butterfly passage increases gradually along the flow line. However, the development of pressure inside the license is 


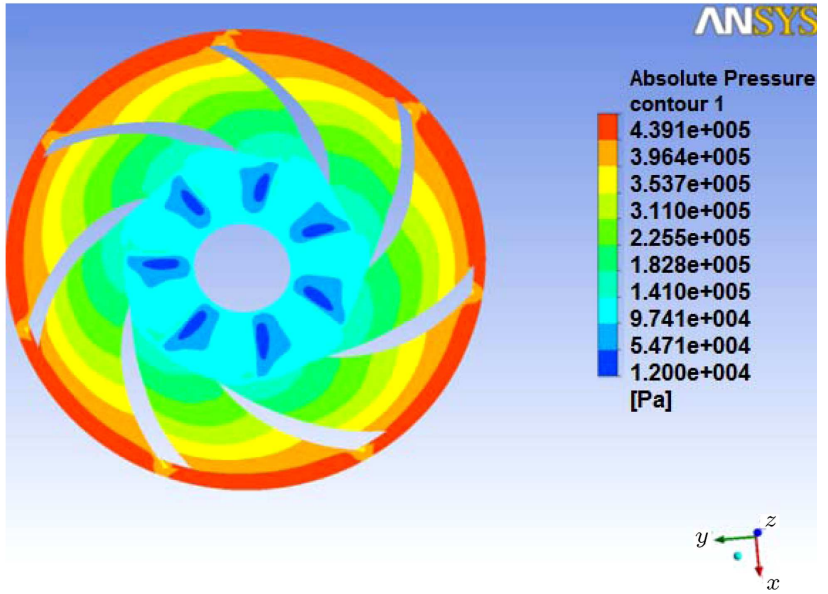

Figure 11. Distribution of pressure on the impeller.

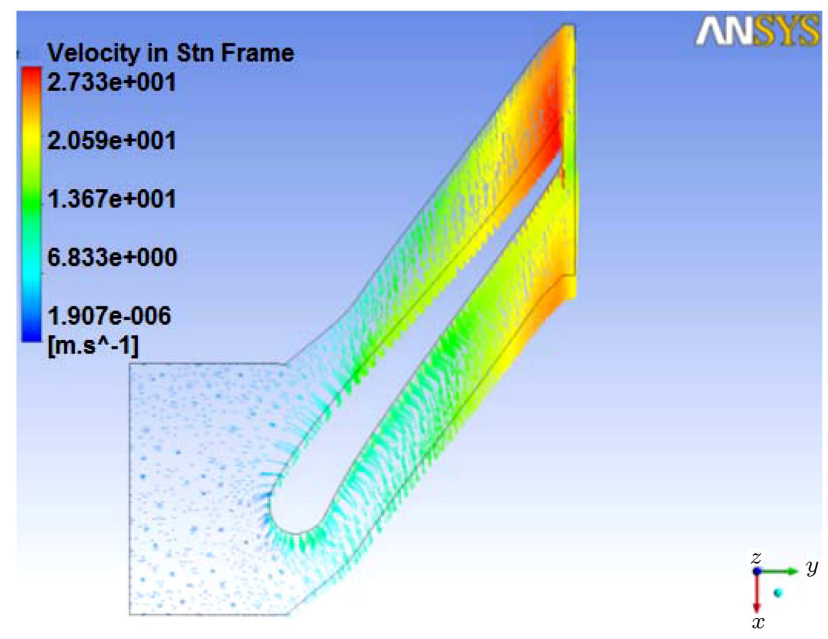

Figure 12. Distribution of speed on the butterfly.

not so uniform. Moreover, the pressure lines are not perpendicular to the surface of the blade pressure inside the passage, which can represent an emphasis on the probability of separating the flow due to the pressure factors. The flow velocity inside the propellant passes along the flow lines gradually, as shown in Figure 12. Moreover, the flow separation is visible at the edge of the blade attack, because the fluid flow at the inlet is not on the blade and is not uniform throughout it. As shown in the figure, the separation of flow occurs on both sides of suction and pressure.

The pressure and speed between the butterfly blades The distribution of the pressure between butterfly blades is shown in Figure 13 . The minimum total pressure appears at the inlet on the butterfly suction surface, and this is the area where cavitation usually occurs. The maximum total pressure value also appears on the impeller output, where the kinetic energy reaches its maximum value.

According to Figure 14, the static pressure dis-

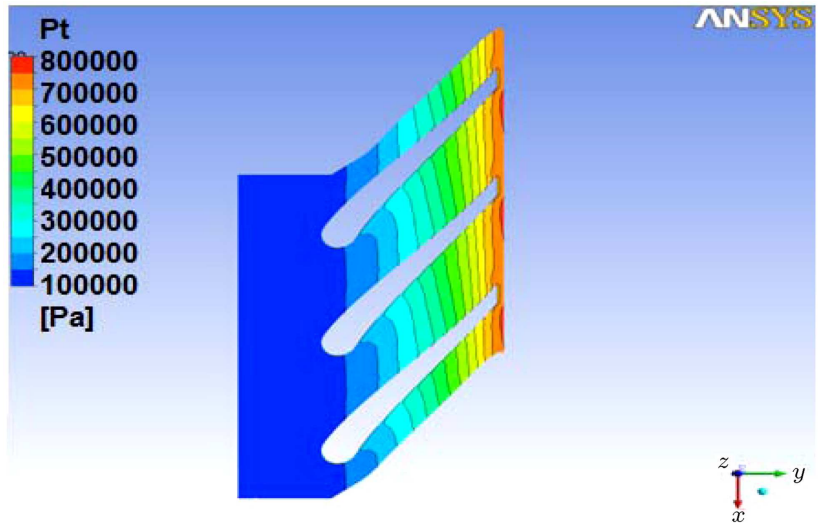

Figure 13. Distribution of total pressure between blades.

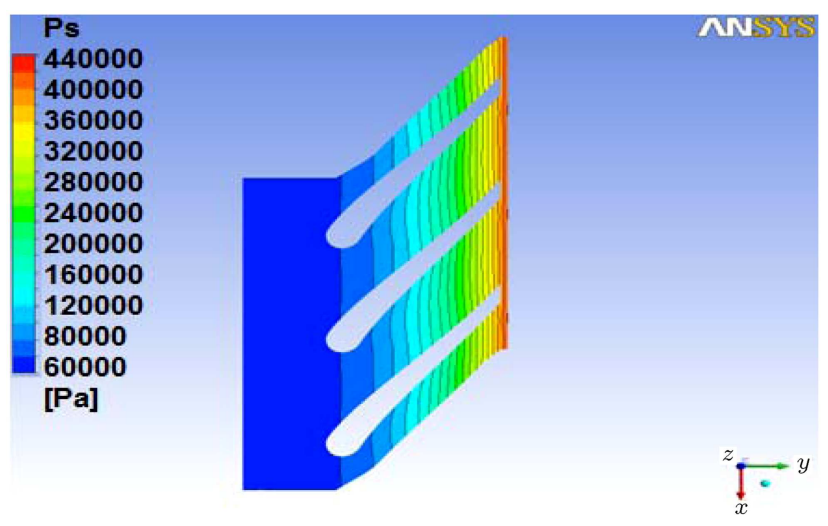

Figure 14. Distribution of static pressure between the blades.

tribution is asymmetric in the impeller blades, and the region with the lowest static pressure is seen behind the blade and suction surface at the entrance. Figure 15 also shows the relative velocity distribution between the propeller blades. Increasing the flow rate from the input to the output reaches its maximum in the blade output. In order to add energy to the fluid (pressurizing it) at the edge of the pressure attack, more pressure is needed on the suction surface. The apparent impact of such pressure distribution is that relative velocities near the suction surface are higher than those at near-surface pressure levels.

Stream pattern at the edge of the attack and escape The effects of the flow of fluid at the edge of the attack and escape in the underlined shapes are given. At least, the pressure is observed at the suction edge of the attack (see Figure 16). Similarly, the variation in the relative velocity of the edge of the attack is observed in Figure 16, which is also due to the separation of the flow that occurs at the edge of the attack, coinciding with the suction side. Similarly, Figure 17 shows variations at the edge of the escape. Figure 18 also depicts the flow lines at the edge of the attack, illustrating how the propeller blades are driven by the fluid. 

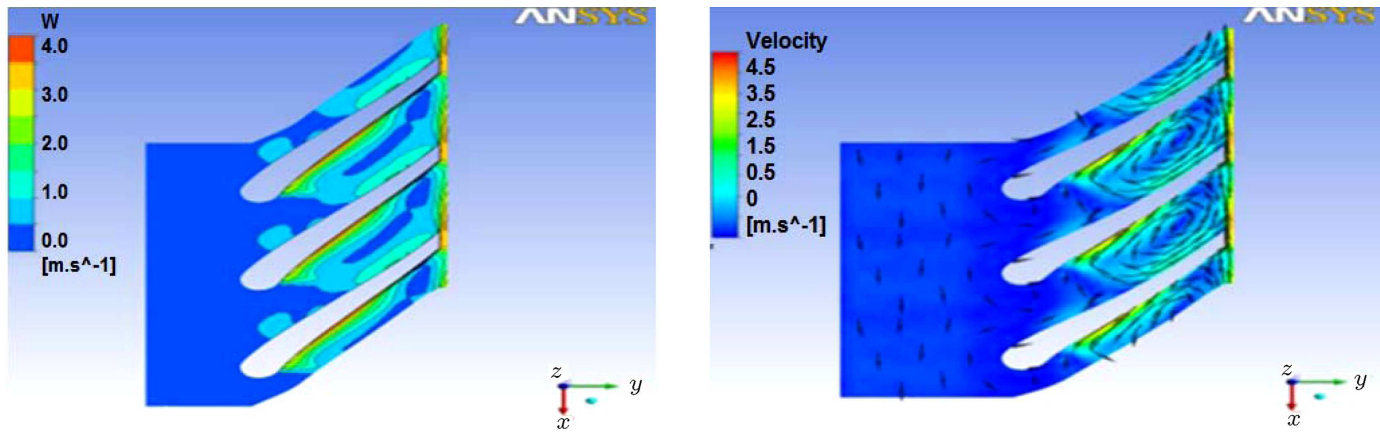

Figure 15. Distribution and relative velocity vector between propeller blades.
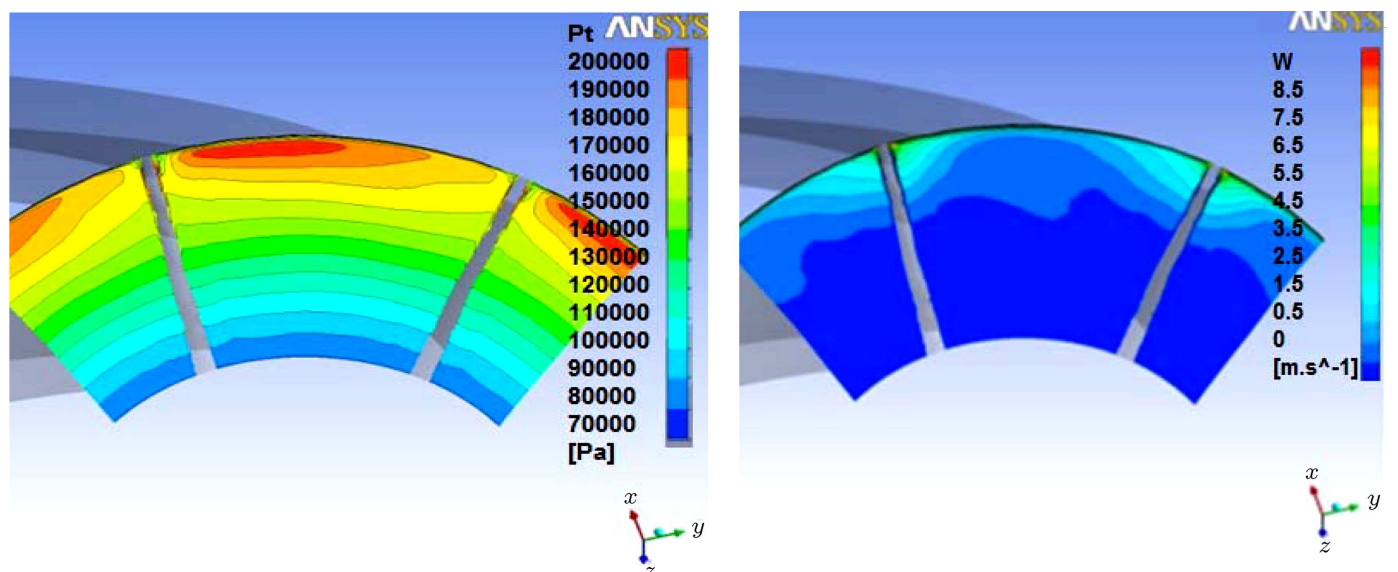

Figure 16. Distribution of total pressure and relative velocity at the edge of the attack.
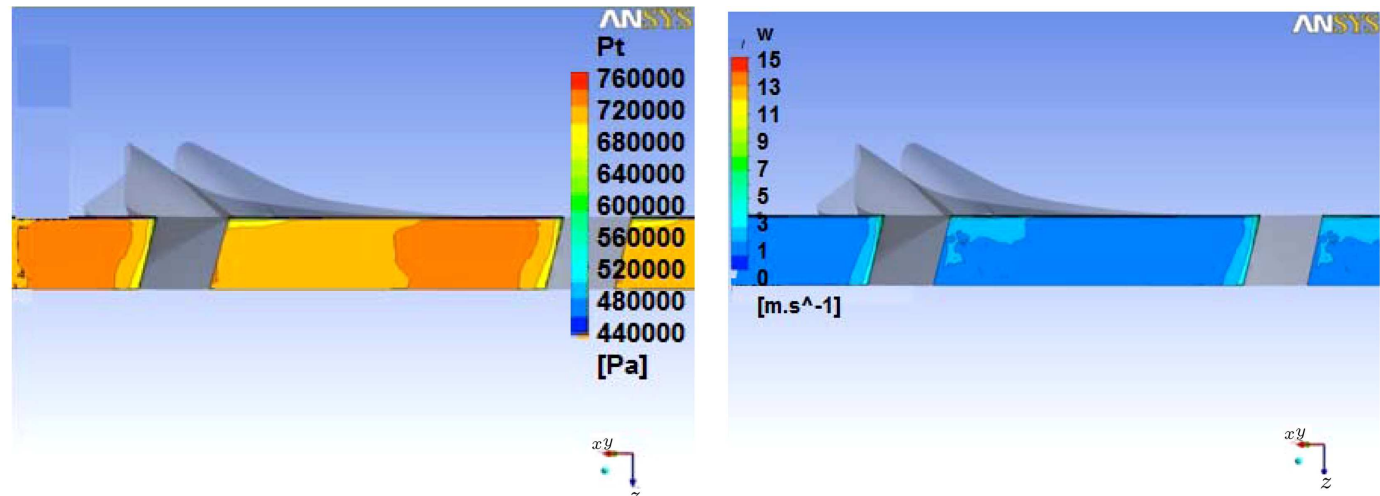

Figure 17. Distribution of total pressure and relative velocity at the edge of the escape.

\subsubsection{Results of cavitation flow simulation}

In order to better illustrate the occurrence of the cavitation phenomenon, the results of this section concerning the two faces for the impeller and the blade-toblade view are expressed in terms of the total pressure parameters and the volume fraction rate. Further to this, for a detailed understanding of the process and the quantification of the point at the beginning of the cavitation, the curve H-NPSH is plotted.

Vapor fraction contour is compared at different pressures, as shown in Figure 19. It can be seen that when the inlet and local pressures of the fluid reduce to less than the vapor pressure, the vapor bubbles are formed along the waiting side at the edge of the inlet of the blade on the suction side, and filling the passage causes the pump to drop and its flow to fluctuate. It is observed that by decreasing the NPSH, the volume fraction of the vapor increases and, in some cases, similar to the fourth state, it completely obstructs the butterfly passage, which is developed in this state of cavitation and is called failure mode.

The contour of the pressure distribution in the blade-to-blade view is shown in Figure 20. In general, Figure 20 shows the following: 
- The pressure level on the blade pressure side is higher than the suction pressure level;

- Pressure at the edge of the pressure side is greater than that at the edge of the suction side;

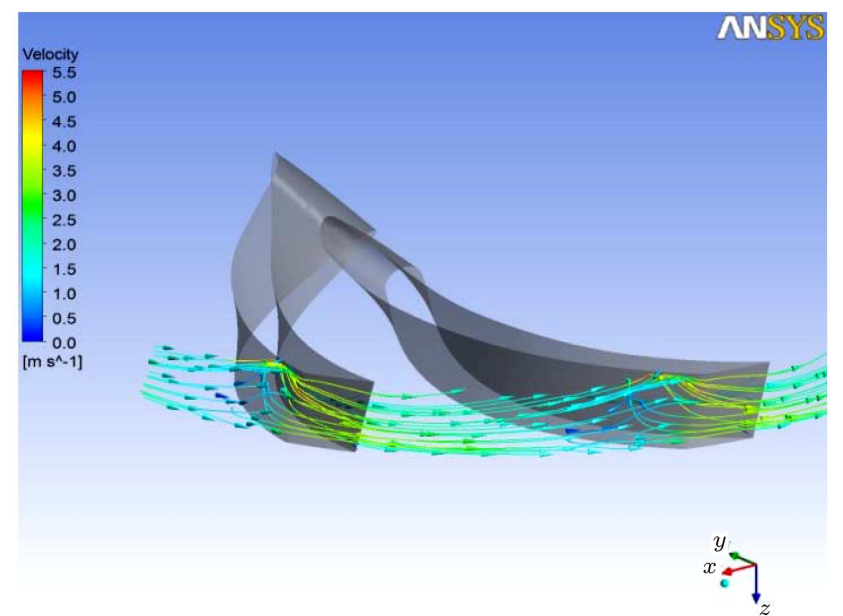

Figure 18. Distribution of speed flow lines at the edge of the escape.
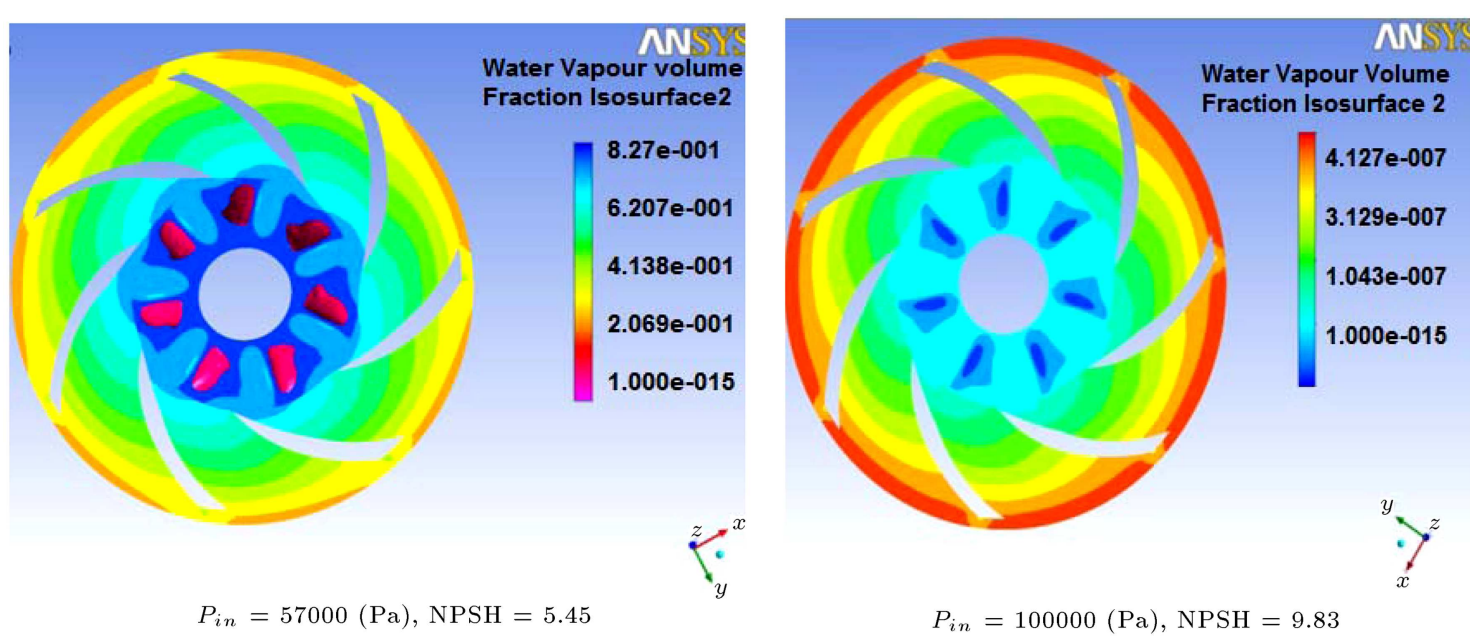

$P_{i n}=100000(\mathrm{~Pa}), \mathrm{NPSH}=9.83$

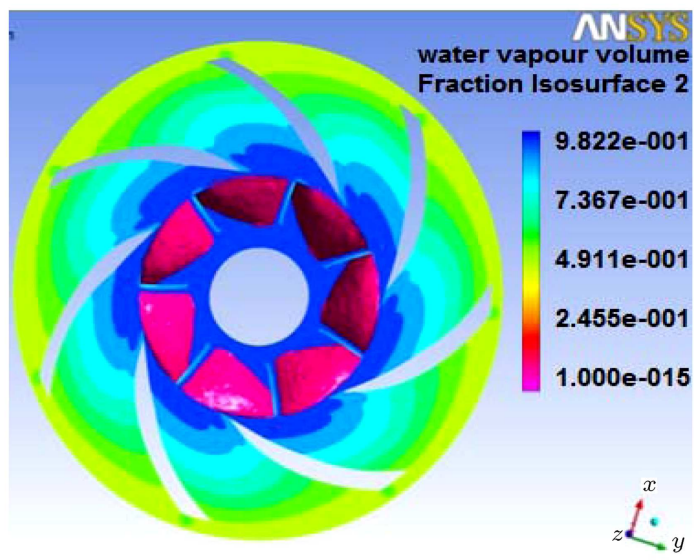

$P_{i n}=42000(\mathrm{~Pa}), \mathrm{NPSH}=3.92$

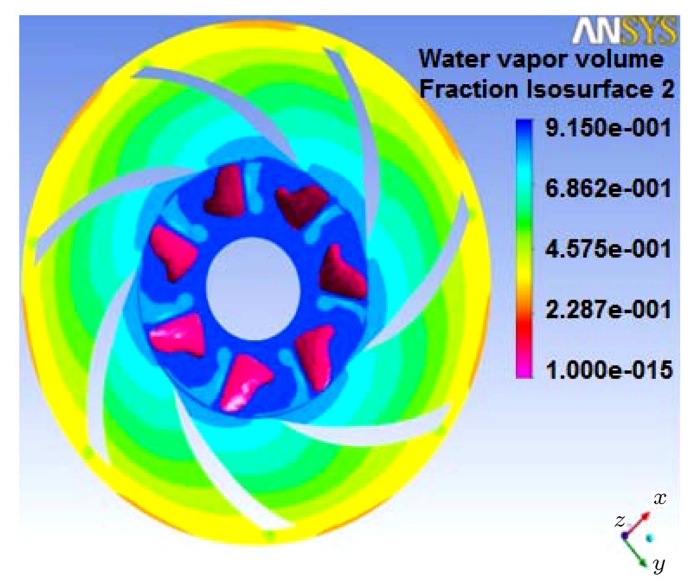

$P_{i n}=45000(\mathrm{~Pa}), \mathrm{NPSH}=4.22$

Figure 19. Volume production rate at the entrance and passage of the butterfly.

- With reduced inlet pressure and NPSH, low-pressure and cavitation-prone areas move from the edge of the suction side to the edge of the attack on the pressure side.

\section{Conclusions}

A numerical model of the propeller was successfully produced, and the complex flow was analyzed using ANSYS CFX software in single-phase and cavitation flow conditions using the model. The simulation results of flow in single-phase mode demonstrated that the internal flow at the suction and blade pressure levels was not uniform due to the tensile flow. The reason for this tangible tension was the separation of the flow at the edge of the attack. The pressure along the lines of the flow gradually increased, and the areas with the maximum amount of pressure on the butterfly's exits were shown. The results of the experiment in non-cavitation flow indicated that the point of greatest efficiency was close to the design point, where $Q=320 \mathrm{~m}^{3} / \mathrm{h}$ and $H=77.33 \mathrm{~m}$. The 

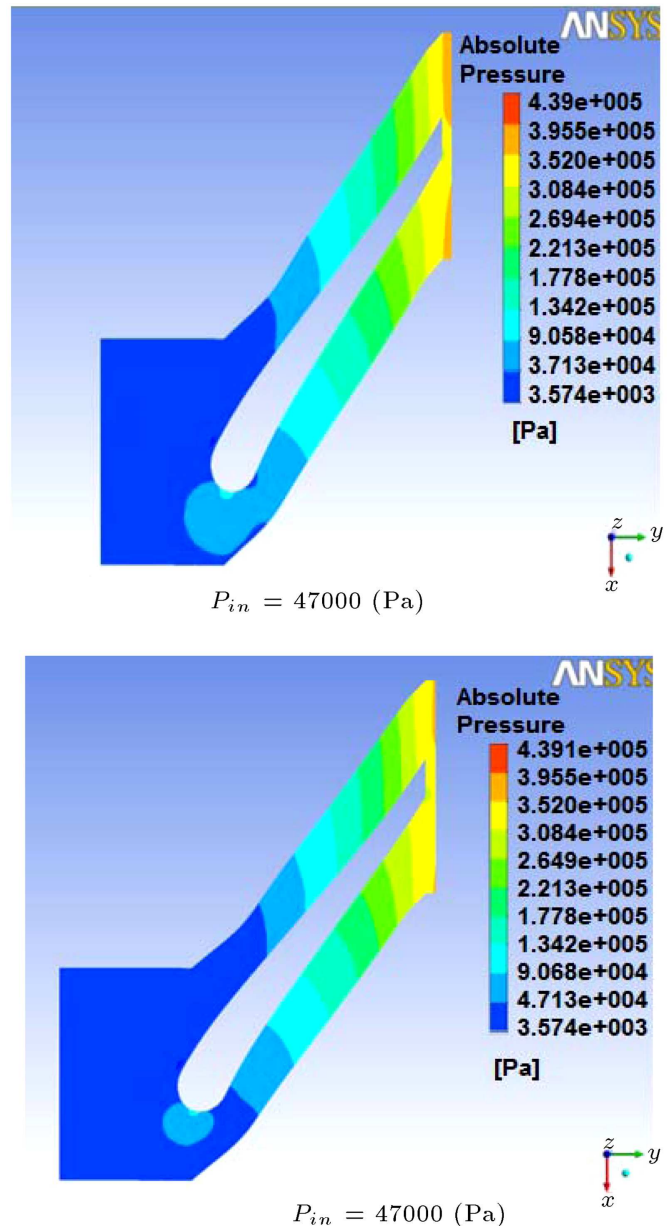
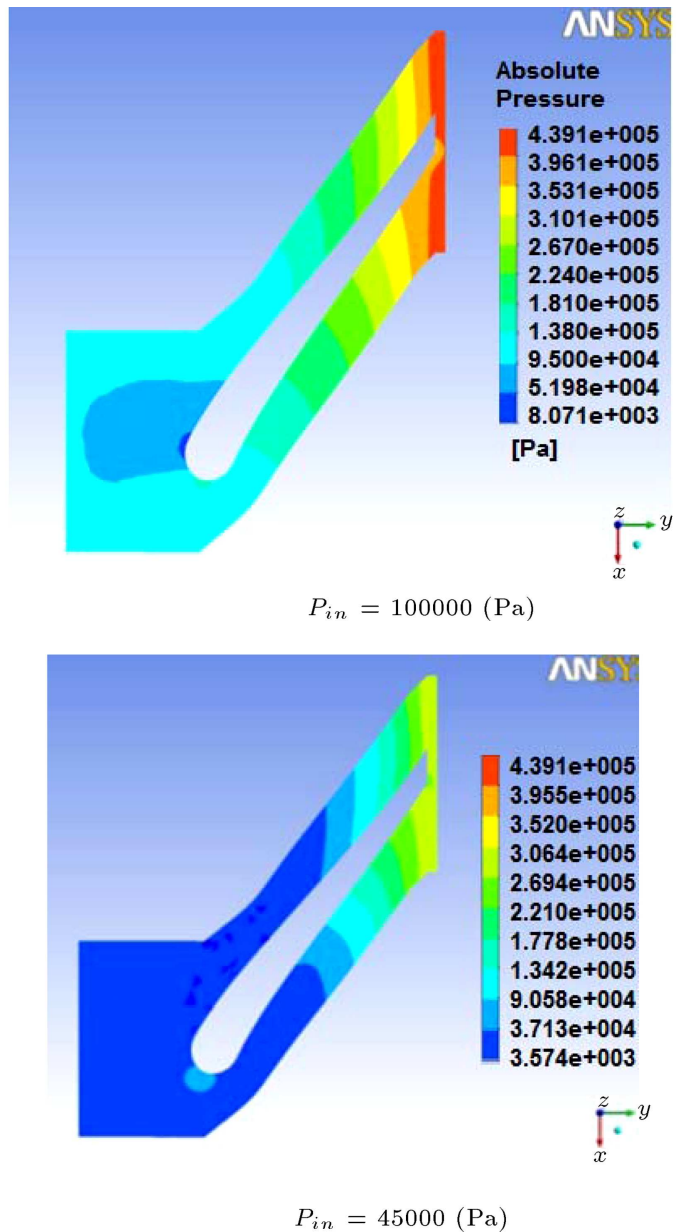

Figure 20. Total pressure distribution field in the passage.

result of the numerical analysis of the cavitation flow was $43.06 \mathrm{~m}$, which was well in agreement with the measured value of 41 . The reason for the difference in head values was that the numerical simulation of the walls was considered to be smooth. However, in reality, there was a friction loss due to the roughness of surfaces. In order to verify the cavitation profile of the pump, different conditions were calculated from the variable inlet pressure from 103,000 $\mathrm{Pa}$ to 42,000 $\mathrm{Pa}$, and it was observed that the volume fraction of the steam increased with decreasing inlet pressure. Irrespective of the pressure level, it always produces cavitation at the back of the edge of the attack. A fraction of the vapor can describe the formation of the flow and the transformation of the bubbles. The cavitation occurred at the back of the edge of the butterfly's attack where the bubbles were formed, while the induced injuries were at the downstream when the bubbles steam occurred due to high pressure around it. Further, for the cavitation flow, the H-NPSHav curve was plotted at $330 \mathrm{~m}^{3} / \mathrm{h}$ and in $1450 \mathrm{rpm}$, as compared to the experimental curve measured. The result showed that, by decreasing the suction energy (NPSHav), the head remained constant until when it reached a value that would allow the determination of vapor bubbles in the NPSHav. The value of NPSHav was equal to the NPSHreq value, which dropped by $3 \%$. The estimated NPSHreq was $3.94 \mathrm{~m}$, while the measured value was $3.76 \mathrm{~m}$, which is a good match. According to the numerical analysis, the most important areas for the occurrence of cavitation for the impeller were the location between the surface of the blade suction and the upper cover plate. The other area was exactly behind the edge of the attack on the pressure side. The result of numerical simulation is $4.7 \%$ of the measured value deviation, which is acceptable accuracy. The origin of this deviation is as follows:

1. The location of the software input may not be the same as the monitoring point in the test equipment.

2. Heat transfer in the numerical analysis is neglected, while the heat generated results from friction and peripheral environment.

\subsection{Suggestions}

Based on the research findings, the following are suggested for future works:

- Numerical flow simulation of the pump inside a 
complete geometry including screw flap and inlet pipe;

- The application of a pump with a transparent body to observe the process of bubble formation in the butterfly passages;

- Investigation of other cavitation visualization methods such as noise and vibration measurement;

- Calculation of the flow field for the two-fold mode;

- Calculation of the flow field for slurry fluid such as hydrocarbons;

- Investigation of the effect of increasing or decreasing the number of blades on the cavitation specification.

\section{References}

1. Tao, R., Xiao, R., Wang, F., and Liu, W. "Cavitation behavior study in the pump mode of a reversible pumpturbine", Renewable Energy, 125, pp. 655-667 (2018).

2. Long, X., Wang, J., Zhang, J., Ji, B. "Experimental investigation of the cavitation characteristics of jet pump cavitation reactors with special emphasis on negative flow ratios" , Experimental Thermal and Fluid Science, 96, pp. 33-42 (2018).

3. Hao, Y. and Tan, L. "Symmetrical and unsymmetrical tip clearances on cavitation performance and radial force of a mixed flow pump as turbine at pump mode", Enewable Energy, 127, pp. 368-376 (2018).

4. Shervani-Tabar, M., Ettefagh, M., Lotfan, S., and Safarzadeh, H. "Cavitation intensity monitoring in an axial flow pump based on vibration signals using multi-class support vector machine", Proceedings of the Institution of Mechanical Engineers, Part C: Journal of Mechanical Engineering Science, 232(17) pp. 30133026 (2017).

5. Lu, X., Wang, D., Shen, W., Zhu, C., and Chen, M. "Experimental investigation on the performance of improving jet pump cavitation with air suction", International Journal of Heat and Mass Transfer, 88, pp. 379-387 (2018).

6. Kumar S. and Deekshith, P. "Exploring cavitation phenomenon with and without ultrasonice transducer", Elsevier, Procedia Engineering, 38, pp. 154164 (2012).

7. Jim Kim, M. and Jin, H. A Study on Prediction of Cavitation for Centrifugal Pump, World Academy of Science and Engineering, pp. 648-655 (2012).

8. Hedi, L. and Hatem, K., Numerical Simulation of Cavitation Flow in Centrifugal Pump, Science Academy of Renewable Energy, 2, pp. 179-185 (2012).

9. Li, P., Huang, Y.F., and Li, J. "Cavitation simulation and NPSH prediction of a double suction centrifugal pump", In IOP Conference Series: Earth and Environmental Science IOP Publishing, 15(6), p. 062025 (2012).
10. Shervani-Tabar, M.T. and Rouhollahi, R. "Numerical study on the effect of the concave rigid boundaries on the cavitation intensity", Scientia Iranica, 24(4), pp. 1958-1965 (2017).

11. Pierrat, D., Gros, L., Pintrand, G., Le Fur, B., and Gyomlai, P. "Experimental and numerical investigations of leading edge cavitation in a helicocentrifugal pump", In The 12th International Symposium of Transport Phenomena and Dynamics on Rotating Machinery, Honolulu, HI, pp. 17-22 (2008).

12. Nohmi, M. and Goto, A. "Cavitation CFD in a centrifugal pump", 5th International Symposium on Cavitation, Osaka (2003).

13. Bagheri, M.R., Seif, M.S., Mehdigholi, H., and Yaakob, O. "Analysis of hydrodynamics and noise prediction of the marine propellers under cavitating and noncavitating conditions", Scientica Iranica, 22(5) pp. 1918-1930 (2015).

14. Safikhani, H. "Sensitivity analysis of the effective centrifugal pump parameters using the EFAST method", Scientica Iranica, Trans. B, 26(1),pp. 421-427 (2019).

15. Qazani, M.R.C., Pedrammehr, S., and Nategh, M.J. "An investigation on the motion error of machine tools' Hexapod table", M.J. Int. J. Precis. Eng. Manuf, 19(463), pp. 45-53 (2018). https://doi.org/10.1007/s12541-018-0056 -5

16. Bavil, A.K. and Razavi, S.E. "On the thermo-flow behavior in a rectangular channel with skewed circular ribs", Mechanics and Industry, 18(2), p. 225 (2017).

\section{Biographies}

Behzad Ranjbar received BS and MS degrees in Mechanical Engineering from the University of Tabriz by researching the pump performance and prevention of their damage by the CFD.

Mirbiouk Ehghaghi is an Associate Professor at University of Tabriz in Mechanical Engineering. He received his $\mathrm{PhD}$ in Mechanical Engineering from University of Tabriz in 2010. His research interest includes turbomachine, computational fluid dynamics, and pumps. He is the Director of the Novin-pump Company in Iran as the leading industry in the pumps.

Seyed Faramarz Ranjbar is an Assistance Professor at the Mechanical Engineering Department at University of Tabriz. He received his PhD in Mechanical Engineering in 2009 from University of Tabriz in the Energy and Fluid Dynamic Field. His research interests include applied heat engineering (heat exchangers design, power plants, turbomachinery, jet engine and gas turbine, internal combustion engines, diesel engine test cell design, heat transfer, industrial plants and installation, and simulation and optimization of thermal systems). 